\title{
Coverings of 3-manifolds by three open solid tori
}

\author{
J. C. Gómez-Larrañaga* F. González-Acuña*† \\ Wolfgang H. Heil ${ }^{\ddagger}$
}

\section{Introduction}

We consider the problem of obtaining a list of compact 3-manifolds that can be obtained as a union of given sets. The concept of an $\mathcal{A}$-category of a manifold $M^{n}$ as introduced in $[\mathrm{CP}]$ is a generalization of the Lusternik-Schnirelmann category $\operatorname{cat}\left(M^{n}\right)$. For a fixed closed connected $k$-manifold $A, 0 \leq k \leq n-1$, a subset $B$ in $M^{n}$ is said to be $A$-contractible if there are maps $\varphi: B \longrightarrow A$ and $\alpha: A \longrightarrow M^{n}$ such that the inclusion map $i: B \longrightarrow M$ is homotopic to $\alpha \cdot \varphi$. The $\mathcal{A}$-category $\operatorname{cat}_{A}\left(M^{n}\right)$ of $M^{n}$ is the smallest number of sets, open and $A$-contractible needed to cover $M^{n}$. Thus when $A$ is a point $P$, $\operatorname{cat}_{P}\left(M^{n}\right)=\operatorname{cat}\left(M^{n}\right)$. In the case $A=S^{1}$ it was shown in [GGH2] that the fundamental group of a closed 3-manifold $M$ with $\operatorname{cat}_{S^{1}}(M)=2$ is cyclic and it then follows from Perelman's work [MT] that in this case $M$ is a lens space; hence $M$ can be covered by two open solid tori. As a first step to obtaining a list of all 3-manifolds with $\operatorname{cat}_{S^{1}}(M)=3$ we ask about minimal covers of $M$ by three open sets, each homotopy equivalent to $S^{1}$. In particular we consider covers of $M$ by three open solid tori.

If a closed 3-manifold $M$ can be covered by three open balls, then $M$ is a connected sum of $S^{3}$ and finitely many $S^{2}$-bundles over $S^{1}$. This was first shown by Hempel and McMillan [HM] and a proof of a slight generalization (allowing punctured balls) was given in [GGH1]. These proofs did not use the Poincarè Conjecture. A much shorter proof can be given by using it, since then it suffices to compute the fundamental group of $M$. In [GGH3] we used this approach, applying Perelman's results about the 3-dimensional spherical space-form conjecture, to obtain a classification of all closed 3-manifolds that can be covered by two open balls and one open solid torus or by one open ball and two open solid tori. This method however does not seem to be amenable to a cover by three

\footnotetext{
*Centro de Investigación en Matemáticas, A.P. 402, Guanajuato 36000, Gto. México. jcarlos@cimat.mx

†Instituto de Matemáticas, UNAM, Ciudad Universitaria, 04510 México, D.F. México. fico@math.unam.mx

¥Department of Mathematics, Florida State University, Tallahasee, FL 32306, USA. heil@math.fsu.edu
} 
open solid tori, since it would involve a computation of fundamental groups of Seifert fiber spaces.

In this paper we obtain a classification of all closed 3-manifolds that can be covered by three open solid tori. The main result is

Theorem: If $M$ is a union of three open solid tori then $M$ is homeomorphic to $B \# \tilde{L}(3)$ or to $B \# \tilde{S}(3)$, where $B$ is a connected sum of $S^{3}$ and $S^{2}$-bundles over $S^{1}$ (with any number of $n \geq 0$ factors), $\tilde{L}(3)$ is a connected sum of at most 3 lens spaces, and $\tilde{S}(3)$ is a closed Seifert fiber space with at most 3 exceptional fibers over any (closed) orbit surface.

This Theorem follows from Corollary 9 (d) which is proved in section 5. Our proofs do not use Perelman's results.

In our proofs we replace unions of balls and solid tori by unions that are obtained by attaching two-handles to the balls and solid tori. This leads us to consider unions of punctured balls, punctured solid tori and punctured lens spaces and general position decompositions (defined in section 2). Such a decomposition of a 3-manifold $M$ consists of a cover of $M$ by pl 3-dimensional submanifolds such that the boundaries of any two intersect transversely and the boundaries of any three have no common intersection points. In Theorem 7 (section 4), we obtain a classification of all compact 3-manifolds (with nonempty boundary) that admit a general position decomposition into punctured balls, punctured lens spaces and one or two punctured solid tori. This is used in section 5 to obtain in Theorem 8 a classification of all closed 3-manifolds that admit a general position decomposition into punctured balls, punctured lens spaces and at most three punctured solid tori. Then Corollary 9 gives a classification of all closed 3-manifolds that are a union of three open submanifolds, each an open ball or an open solid torus.

\section{Preliminaries}

The following lemma, proved in [GGH1], Corollary 1(a), allows us to work in the pl-category.

Lemma 1 Suppose $M$ is a closed 3-manifold covered by three open sets $U_{1}$, $U_{2}, U_{3}$, such that $U_{i}$ is homeomorphic to the interior of a compact connected 3-manifold $M_{i}(i=1,2,3)$. Then $M$ admits a covering $M=M_{1} \cup M_{2} \cup M_{3}$ such that $\partial M_{1}$ is transverse to $\partial M_{2}, \partial M_{3} \subset \operatorname{int}\left(M_{1} \cup M_{2}\right)$, and $M_{1}, M_{2}, M_{3}$ are $p l$ embedded.

By an open punctured ball (resp. solid torus) we mean a manifold homeomorphic to an open ball (resp. solid torus) with a finite number of points removed. 
By an ( $n$-times) punctured $M$ we mean a manifold obtained from $M$ by removing interiors of $(n)$ disjoint balls in $\operatorname{int}(M)$. We allow $n=0$. Note that a connected (punctured $M)=M \# H$, for some punctured ball $H$.

We will use the following notations throughout this paper:

For a 3-manifold $M$, the manifold obtained by filling in all 2-sphere boundary components by 3 -balls is denoted by $\hat{M}$.

$H$ or $H_{i}$ denotes a punctured ball with finitely many punctures (with possibly no punctures).

$W$ or $W_{i}$ denotes a handlebody (orientable or non-orientable).

$L$ or $L_{i}$ denotes a (punctured) lens space (possibly $S^{1} \times S^{2}$ or $S^{3}$ ).

$V$ or $V_{i}$ denotes a (punctured) solid torus.

$S(n)$ denotes a Seifert fiber space with at most $n$ exceptional fibers and any compact orbit surface with non-empty boundary.

$\tilde{S}(n)$ denotes a closed Seifert fiber space with at most $n$ exceptional fibers and any (closed) orbit surface.

$W(S(n))$, a Seifert web on $S(n)$, is obtained from $S(n)$ by attaching any finite number of 1-handles.

$\mathbb{B}$ denotes the collection of manifolds that are connected sums of $S^{3}$ and $S^{2}$ bundles over $S^{1}$ (with finitely many factors).

$\mathbb{W}$ denotes the collection of 3 -manifolds $W_{1} \# \cdots \# W_{n}, n \geq 0$ that are connected sums of handlebodies $W_{i}$ (with finitely many factors).

For collections of manifolds $\mathbb{X}, \mathbb{Y}$ we denote by $\mathbb{X} \# \mathbb{Y}$ the collection of manifolds of the form $X \# Y$, where $X \in \mathbb{X}$ and $Y \in \mathbb{Y}$.

For convenience we write $M \in \mathbb{B} \# \hat{L} \# H$ instead of saying that $M$ is a connected sum of $\hat{L}, H$, and a finite number of $S^{2}$-bundles over $S^{1}$.

It is easy to prove (e.g. Lemma 2 of $[\mathrm{GH}]$ ) that the collection $\mathbb{B} \# \mathbb{W} \# H$ is closed under attaching 1-handles, moreover:

Lemma 2 The family $\mathbb{B} \# \mathbb{W} \# L_{1} \cdots \# L_{n} \# W(S(n)) \# H$ is closed under the operation of attaching 1-handles.

Remark 3 (a) Attaching a 2-handle to $L$ results in $L$ (with one more puncture).

(b) Attaching a 2-handle to $V$ results in $V$ (with one more puncture) or in $L$.

(c) Cutting $L$ along a disk results in $L \cup H$ or, if $L$ is a punctured $S^{2} \times S^{1}$, possibly in $H$.

(d) Cutting $V$ along a disk results in $V \cup H$ or $H$.

The following lemma is well known for irreducible 3-manifolds. Noting that for an irreducible 3-manifold $\hat{M}$ every 2-sphere in $M$ bounds a punctured ball in $M$, the proof in [GGH] yields: 
Lemma 4 Suppose $M$ is a compact 3-manifold such that $\hat{M}$ is irreducible.

If $M$ contains a 2-sided compressible torus $T$ then either $T$ bounds a punctured solid torus or a punctured knot space $R$ in $M$ such that a meridian curve of $\partial R$ bounds a disk $D$ in $\overline{M-R}$. In particular if $T$ is a compressible boundary component of $M$ then $M$ is a punctured solid torus.

Let $M_{1}, \cdots, M_{n}$ be compact submanifolds of a 3-manifold $N$. We say that

$$
(*) \quad N=M_{1} \cup \cdots \cup M_{n}
$$

is a general position decomposition of $N$ if $\partial M_{i}$ is transverse to $\partial M_{j}$ for each $i \neq j,(i, j=1, \cdots, n)$, and $\partial M_{i} \cap \partial M_{j} \cap \partial M_{k}=\emptyset$ for all distinct $i, j, k$.

The complexity $\alpha$ of the general position decomposition $(*)$ is the number of components of $\left\{\partial M_{i} \cap \partial M_{j} \mid i \neq j, i, j=1, \cdots, n\right\}$.

Lemma 5 Suppose $N=M_{1} \cup \cdots \cup M_{n}$ is a general position decomposition of $N$ and $N \subset \operatorname{int}(M)$ for some compact 3-manifold $M$. If a component $c$ of $\partial M_{i} \cap \partial M_{j}$ is null homotopic on $\partial M_{i}$ then there is a disk $D$ on $M_{i}$ such that $\partial D=D \cap \partial M_{j}$ for some $i \neq j$ and $D \cap M_{k}=\emptyset$ or $D \subset \operatorname{int}\left(M_{k}\right)$ for all $k \neq i, j$ and either

(i) $N \approx M_{1}^{\prime} \cup \cdots \cup M_{n}^{\prime}$ is a general position decomposition of $N$ with complexity $\alpha^{\prime}<\alpha$, where $M_{k}^{\prime}=M_{k}$ for $k \neq j$ and $M_{j}^{\prime}$ is obtained from $M_{j}$ by attaching a 2-handle to $\partial M_{j}$ with cocore $D$, or

(ii) $N=N^{\prime}$ or $N$ is obtained from $N^{\prime}$ by attaching a 1-handle to $\partial N^{\prime}$, where $N^{\prime} \approx M_{1}^{\prime} \cup \cdots \cup M_{n}^{\prime}$ is a general position decomposition with complexity $\alpha^{\prime}<\alpha$, and where $M_{k}^{\prime}=M_{k}$ for $k \neq j$ and $M_{j}^{\prime}=M_{j} \backslash D$.

(Here $M_{j} \backslash D$ is obtained from $M$ by cutting along the properly embedded disk $D)$.

\section{Proof.}

The component $c$ bounds a disk on $\partial M_{i}$. Let $D$ be an innermost such disk on $\partial M_{i}$, i.e. $\partial D=D \cap \partial M_{j}$ for some $j \neq i$ and $D \cap M_{k}=\emptyset$ or $D \subset \operatorname{int}\left(M_{k}\right)$ for all $k \neq i, j$. Let $U(D)$ be a regular neighborhood $\left(\operatorname{rel} \partial M_{j}\right)$ of $D$ in $M$.

If $D$ is not contained in $M_{j}$ let $M_{j}^{\prime}=M_{j} \cup U(D)$ (If $D \subset \operatorname{int}\left(M_{k}\right)$ for $k \neq j$ choose $U(D) \subset \operatorname{int}\left(M_{k}\right)$ ). Then $M_{j}^{\prime}$ and $N$ are as in (i) (see Fig 1).

If $D \subset M_{j}$ let $M_{j}^{\prime}=\overline{M_{j}-U(D)}$. Then $M_{j}^{\prime}$ and $N^{\prime}$ are as in (ii) (see Fig 2). If $D \cap M_{k}=\emptyset$ for all $k \neq i, j$ then $N$ is obtained from $N^{\prime}$ by attaching a 1-handle with cocore $D$. If $U(D) \subset$ int $M_{k}$ for some $k \neq i, j$ then $N=N^{\prime}$. 

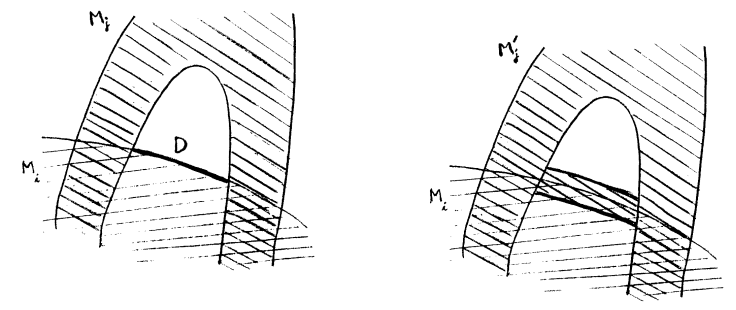

Figure 1:
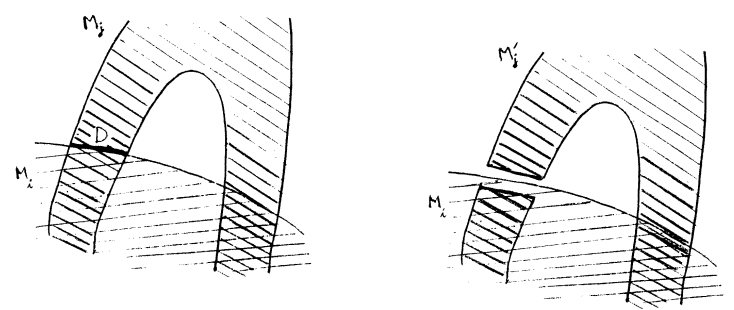

Figure 2:

\section{Complexity 0.}

First we consider general position decompositions of punctured balls, punctured lens spaces and at most 2 punctured solid tori with complexity 0 .

Proposition 6 Let $N \subset$ int $M$ for some 3-manifold $M$.

(a) If $N=\cup_{i} H_{i} \cup L_{1} \cdots \cup L_{n}$ is a general position decomposition with complexity 0 then $N \in \mathbb{B} \# \hat{L}_{1} \# \cdots \# \hat{L}_{n} \# H$.

(b) If $N=\cup_{i} H_{i} \cup L_{1} \cdots \cup L_{n} \cup V_{1}$ is a general position decomposition with complexity 0 then $N \in \mathbb{B} \# \hat{L}_{1} \# \cdots \# \hat{L}_{n} \# H$ or $N \in \mathbb{B} \# \hat{L}_{1} \# \cdots \# \hat{L}_{n} \# \hat{V}_{1} \# H$.

(c) If $N=\cup_{i} H_{i} \cup L_{1} \cdots \cup L_{n} \cup V_{1} \cup V_{2}$ is a general position decomposition with complexity 0 then

$N \in \mathbb{B} \# \hat{L}_{1} \# \cdots \# \hat{L}_{n} \# \hat{V}_{i} \# H(i=1$ or 2$)$ or

$N \in \mathbb{B} \# \hat{L}_{1} \# \cdots \# \hat{L}_{n} \# \hat{V}_{1} \# \hat{V}_{2} \# H$ or

$N \in \mathbb{B} \# \hat{L}_{1} \# \cdots \# \hat{L}_{n} \# \hat{L} \# H$.

(Recall that we allow $\hat{L}=S^{3}$ ).

\section{Proof.}

Let $T_{i}$ denote the torus boundary of $V_{i}$ and let $\mathbf{S}$ denote the collection of 2-spheres $\bigcup_{i} \partial H_{i} \cup \partial L_{1} \cdots \cup \partial L_{n}$, resp. $\bigcup_{i} \partial H_{i} \cup \partial L_{1} \cdots \cup \partial L_{n} \cup\left(\partial V_{1}-T_{1}\right)$, resp. $\bigcup_{i} \partial H_{i} \cup \partial L_{1} \cdots \cup \partial L_{n} \cup\left(\partial V_{1}-T_{1}\right) \cup\left(\partial V_{2}-T_{2}\right)$. For each $i, \mathbf{S} \cap$ int $H_{i}$ 
cuts $H_{i}$ into punctured balls. Similarly, since $\hat{V}_{i}$ is irreducible, $\mathbf{S} \cap V_{i}$ cuts $V_{i}$ into a punctured solid torus $V_{i}^{\prime}$ and punctured balls. Also $\mathbf{S} \cap L_{j}$ cuts $L_{j}$ into a punctured lens space $L_{j}^{\prime}$ and punctured balls. (If $L_{j}$ is a punctured $S^{2} \times S^{1}$ then $L_{j}^{\prime}$ may be a punctured $S^{2} \times S^{1}$ or a punctured ball; in any other case $\hat{L}_{j}^{\prime}=\hat{L}_{j}$ ). Denote the collection of all the resulting punctured balls by $\left\{H_{k}^{\prime}\right\}$.

(a) In this case $N=\bigcup_{k} H_{k}^{\prime} \cup L_{1}^{\prime} \cdots \cup L_{n}^{\prime}$ is obtained from a disjoint collection of punctured balls $H_{k}^{\prime}$ and punctured lens spaces $L_{j}^{\prime}$ by identifying some boundary components in pairs and the result follows.

(b) In this case consider $N=\bigcup_{k} H_{k}^{\prime} \cup L_{1}^{\prime} \cdots \cup L_{n}^{\prime} \cup V_{1}^{\prime}$. If there is a point $x$ in $\operatorname{int}\left(H_{k}^{\prime}\right) \cap V_{1}^{\prime}$ then (viewing $x$ in $V_{1}^{\prime}$ ) each point $y$ in $V_{1}^{\prime}$ can be joined to $x$ by a path $w$ in $V_{1}^{\prime}$ such that $\operatorname{int}(w) \subset \operatorname{int}\left(V_{1}^{\prime}\right)$, i.e. $w$ does not cross $\partial H_{k}^{\prime}$. Hence (viewing $x$ in $H_{k}^{\prime}$ ), $w$ lies in $H_{k}^{\prime}$ and therefore $V_{1}^{\prime} \subset H_{k}^{\prime}$. Similarly, if $V_{1}^{\prime} \cap$ int $L_{j}^{\prime} \neq \emptyset$ we obtain $V_{1}^{\prime} \subset L_{j}^{\prime}$. In these cases we delete $V_{1}^{\prime}$ from the decomposition of $N$ and obtain $N$ as in case (a).

In any other case $N$ is obtained from the disjoint collection of the punctured balls $H_{k}^{\prime}$, the punctured lens spaces $L_{j}^{\prime}$, and the punctured solid torus $V_{1}^{\prime}$ by identifying some boundary spheres in pairs. The result follows.

(c) We have $N=\bigcup_{k} H_{k}^{\prime} \cup L_{1}^{\prime} \cdots \cup L_{n}^{\prime} \cup V_{1}^{\prime} \cup V_{2}^{\prime}$.

case (c1): $T_{1}$ is not contained in $i n t V_{2}^{\prime}$.

By the argument above, considering paths in $V_{2}^{\prime}$, we see that if $V_{2}^{\prime} \cap H_{i}^{\prime} \neq \emptyset$, resp. $V_{2}^{\prime} \cap L_{i}^{\prime} \neq \emptyset$, resp. $V_{2}^{\prime} \cap V_{1}^{\prime} \neq \emptyset$, then $V_{2}^{\prime} \subset H_{i}^{\prime}$, resp. $V_{2}^{\prime} \subset L_{i}^{\prime}$, resp. $V_{2}^{\prime} \subset V_{1}^{\prime}$. We delete $V_{2}^{\prime}$ from the decomposition of $N$ and get $N$ as in (b) (the third term in the list $6(\mathrm{c})$ ).

If $V_{2}^{\prime}$ is disjoint from $H_{i}^{\prime}, L_{i}^{\prime}$, and $V_{1}^{\prime}$ we obtain $N$ from $V_{2}^{\prime}$ and $N^{\prime}=\bigcup_{k} H_{k}^{\prime} \cup$ $L_{1}^{\prime} \cdots \cup L_{n}^{\prime} \cup V_{1}^{\prime}$ by identifying 2 -spheres in the boundary. Since $N^{\prime}$ is as in (b), $N$ is as in the second and third term of $6(\mathrm{c})$.

The same argument applies if $T_{2}$ is not contained in int $V_{1}^{\prime}$.

case (c2): $T_{1} \subset$ int $V_{2}^{\prime}$ and $T_{2} \subset$ int $V_{1}^{\prime}$

Then by [ (3.1)(b) of [GGH] ] we have $\hat{V}_{1}^{\prime} \cup \hat{V}_{2}^{\prime}=\hat{L}$ and $N=\bigcup_{k} H_{k}^{\prime} \cup L_{1}^{\prime} \cdots \cup$ $L_{n}^{\prime} \cup L$ is as in case (a) with one more lens space summand, which yields the third term of $6(\mathrm{c})$.

\section{Balls, lens spaces, and two solid tori.}

We now consider the case when the complexity is strictly positive.

Theorem 7 Let $N \subset$ int $M$ for some 3-manifold $M$. 
(a) If $N=\bigcup_{i} H_{i} \cup L_{1} \cdots \cup L_{n}$ is a general position decomposition then $N \in$ $\mathbb{B} \# \mathbb{W} \# \hat{L}_{1} \# \cdots \# \hat{L}_{n} \# H$.

(b) If $N=\bigcup_{i} H_{i} \cup L_{1} \cdots \cup L_{n} \cup V_{1}$ is a general position decomposition then $N \in \mathbb{B} \# \mathbb{W} \# \hat{L}_{1} \# \cdots \# \hat{L}_{n} \# \hat{L}_{n+1} \# H$.

(c) If $N=\bigcup_{i} H_{i} \cup L_{1} \cdots \cup L_{n} \cup V_{1} \cup V_{2}$ is a general position decomposition then

$\left(c_{1}\right) N \in \mathbb{B} \# \mathbb{W} \# \hat{L}_{1} \# \cdots \# \hat{L}_{n} \# \hat{L}_{n+1} \# \hat{L}_{n+2} \# H$ or

$\left(c_{2}\right) N \in \mathbb{B} \# \mathbb{W} \# \hat{L}_{1} \# \cdots \# \hat{L}_{n} \# W(S(2)) \# H$.

(Recall that we allow $\hat{L}_{k}=S^{3}$ ).

\section{Proof.}

The proof is by induction on the complexity $\alpha$ of the decomposition for $N$. If $\alpha=0$ we obtain $N$ as in Proposition 6 with $\mathbb{W}=\emptyset$ or $\mathbb{W}=\hat{V}_{i}$ or $\mathbb{W}=\hat{V}_{1} \# \hat{V}_{2}$. Suppose $\alpha \geq 1$.

cases (a) and (b):

Let $\mathbf{C}$ denote the collection of the circles $\left\{\partial H_{i} \cap \partial H_{j}(i \neq j), \partial H_{i} \cap \partial L_{k}\right.$, $\left.\partial L_{i} \cap \partial L_{j}\right\}$, resp. $\left\{\partial H_{i} \cap \partial H_{j}(i \neq j), \partial H_{i} \cap \partial L_{k}, \partial L_{i} \cap \partial L_{j}, \partial H_{i} \cap \partial V_{1}\right.$, $\left.\partial L_{i} \cap \partial V_{1}\right\}$ and consider a circle $c$ of $\mathbf{C}$ on $\partial H_{i}$ (resp. $\partial L_{i}$ ). Since $c$ is null homotopic on $\partial H_{i}$ (resp. $\partial L_{i}$ ) we apply Lemma 5 .

In case (i) of Lemma 5 we obtain a new general position decomposition of $N$ with smaller complexity that differs from the given one only in that some $H_{j}$ or $L_{j}$ or $V_{1}$ is replaced by adding a 2 -handle. By Remark 3 the new decomposition is of the same type, where $V_{1}$ either survives or is replaced by $L_{n+1}$, and the Theorem follows by induction. (If $V_{1}$ survives to the end, it becomes a part of $\mathbb{W})$.

In case (ii) we obtain $N$ from $N^{\prime}$ by attaching a 1-handle, where $N^{\prime}$ has a general position decomposition of complexity $<\alpha$ and is as in (a) or (b) except that some $H_{j}$ or $L_{j}$ or $V_{1}$ is cut along a disk. By Remark $3, N^{\prime}$ is as in (a) or (b) and the result follows by induction and Lemma 2 .

case $(\mathrm{c})$ :

Let $T_{i}$ be the torus boundary of $V_{i}$.

If there is a component of $T_{1} \cap T_{2}$ that is null homotopic on $T_{1}$ or $T_{2}$, or if $T_{1} \cap T_{2}=\emptyset$, then we apply the same argument as above (using Lemma 5 ) to obtain $N$ from $N^{\prime}$ by attaching 1-handles and where $N^{\prime}$ has a decomposition of the same type as $N$ and with smaller complexity.

Thus assume that $T_{1} \cap T_{2} \neq \emptyset$ and every component of $T_{1} \cap T_{2}$ is not null homotopic on $T_{1}$ and $T_{2}$. Then the components of $\partial V_{1} \cap V_{2}$, resp. $\partial V_{2} \cap V_{1}$, consist of annuli $A_{i}$, resp. $A_{j}^{\prime}$. 
If a boundary component of an $A_{j}^{\prime}$, say, is null homotopic in $V_{1}$ then all the $A_{j}^{\prime}$ are compressible in $V_{1}$ and we find a meridian disk $D$ of $V_{1}$ that is disjoint to all $A_{j}^{\prime}$ and such that $\partial D$ is disjoint to $H_{i}$ (resp. $L_{j}$ ) or $D \subset \operatorname{int}\left(H_{i}\right)$ (resp. $\left.D \subset \operatorname{int}\left(L_{j}\right)\right)$. A regular neighborhood of $D$ in $V_{1}$ decomposes $V_{1}$ into two punctured balls $H_{0}, H_{0}^{\prime}$ and we replace $V_{1} \cup V_{2}$ in the decomposition of $N$ by $H_{0} \cup H_{0}^{\prime} \cup V_{2}$ to obtain $N$ as in case (b). This results in $7\left(c_{1}\right)$.

Thus we may assume that the boundary components of $A_{j}^{\prime}$, resp. $A_{i}$, are not null homotopic in $V_{1}$, resp. $V_{2}$ and it follows that the $A_{j}^{\prime}$ are incompressible boundary parralel annuli in $\hat{V}_{1}$ (see for example [Ha]) and there is a Seifert fibration of $\hat{V}_{1}$ such that the $A_{i}$ 's and $A_{j}^{\prime}$ 's consist of fibers. The fibration of $\hat{V}_{1} \cap \hat{V}_{2}$ extends to a Seifert fibration of $\hat{V}_{2}$ and hence $\hat{V}_{1} \cup \hat{V}_{2}=S(2)$. The result follows.

\section{Closed 3-manifolds and three solid tori.}

The main result (Corollary 9) follows from the following

Theorem 8 Let $M$ be a closed 3-manifold.

(a) If $M$ admits a general position decomposition $M=\bigcup_{i} H_{i} \cup L_{1} \cdots \cup L_{l}$ then $M \in \mathbb{B} \# \hat{L}_{1} \# \cdots \# \hat{L}_{l}$.

(b) If $M$ admits a general position decomposition $M=\bigcup_{i} H_{i} \cup L_{1} \cdots \cup L_{l} \cup V_{1}$ then $M \in \mathbb{B} \# \hat{L}_{1} \# \cdots \# \hat{L}_{l} \# \hat{L}_{l+1}$.

(c) If $M$ admits a general position decomposition $M \in \bigcup_{i} H_{i} \cup L_{1} \cdots \cup L_{l} \cup V_{1} \cup V_{2}$ then $M \in \mathbb{B} \# \hat{L}_{1} \# \cdots \# \hat{L}_{l} \# \hat{L}_{l+1} \# \hat{L}_{l+2}$.

(d) If $M$ admits a general position decomposition $M=\bigcup_{i} H_{i} \cup L_{1} \cdots \cup L_{l} \cup V_{1} \cup$ $V_{2} \cup V_{3}$ then

$\left(d_{1}\right) M \in \mathbb{B} \# \hat{L}_{1} \# \cdots \# \hat{L}_{l} \# \hat{L}_{l+1} \# \hat{L}_{l+2} \# \hat{L}_{l+3}$ or

$\left(d_{2}\right) M \in \mathbb{B} \# \hat{L}_{1} \# \cdots \# \hat{L}_{l} \# \tilde{S}(3)$.

(Again recall that we allow $\hat{L}_{k}=S^{3}$ ).

Proof.

We only demonstrate (d), since the proofs for (a), (b) and (c) are similar and easier.

Write $M=N \cup V_{3}$, where $N=\bigcup_{i} H_{i} \cup L_{1} \cdots \cup L_{l} \cup V_{1} \cup V_{2}$ is as in Theorem $7\left(c_{1}\right)$ or $\left(c_{2}\right)$.

In case $7\left(c_{1}\right)$ we represent $N$ as

$$
(* *) \quad N=H \cup K_{1} \cup \cdots \cup K_{m} \cup W_{1} \cup \cdots \cup W_{n} \cup L_{1} \cup \cdots \cup L_{l} \cup L_{l+1} \cup L_{l+2}
$$

where $H$ is a punctured ball, $K_{j}$ is a once-punctured $S^{2}$-bundle over $S^{1}, W_{i}$ is a once-punctured handlebody $(\mathrm{j}=1, \ldots, \mathrm{m} ; \mathrm{i}=1, \ldots, \mathrm{n})$, and $L_{k}$ is a once 
punctured lens space $(\mathrm{k}=1, \ldots, \mathrm{l}+2)$. We may assume that $\hat{L_{k}} \neq S^{2} \times S^{1}$ since otherwise it can be considered as a $K_{j}$. Moreover $K_{j} \cap K_{i}=W_{j} \cap W_{i}=$ $K_{j} \cap L_{k}=W_{i} \cap L_{k}=\emptyset$ for $i \neq j$, and $H \cap W_{i}=\partial H \cap \partial W_{i}=C_{i}(i=1, \ldots, n)$, $H \cap K_{j}=\partial H \cap \partial K_{j}=C_{j}^{\prime}(j=1, \ldots, m), H \cap L_{k}=\partial H \cap \partial L_{k}=C_{k}^{\prime \prime}(\mathrm{k}=$ $1, \ldots, 1+2)$ are 2 -spheres. Let $S_{j}$ be a non-separating 2-sphere in $i n t K_{j}$. We may assume that $C_{i}, C_{j}^{\prime}, C_{k}^{\prime \prime}$ and $S_{j}$ are transverse to $\partial V_{3}$. Since $M$ is closed, $\partial V_{3} \subset \operatorname{int}(N)$ and $\partial N \subset \operatorname{int}\left(V_{3}\right)$. In particular, for each $i$, the non-sphere boundary component $F_{i}$ of $W_{i}$ is contained in $\operatorname{int}\left(V_{3}\right)$ and separates $V_{3}$ into two components.

Let $\beta$ denote the number of components of $\mathbf{S}=\partial V_{3} \cap\left(\bigcup_{i} C_{i} \cup \bigcup_{j} C_{j}^{\prime} \cup \bigcup_{j} S_{j} \cup\right.$ $\left.\bigcup_{j} C_{k}^{\prime \prime}\right)$

First assume $\beta=0$.

If for some $i$, no component of $\partial V_{3}$ is contained in $W_{i}$ then each point of $W_{i} \cap V_{3}$ can be joined to a point of $F_{i}$ by a path in $W_{i}$ that misses $\partial V_{3}$; hence this path must lie entirely in $V_{3}$ and it follows that $W_{i} \subset V_{3}$. Deleting $W_{i}$ from $(* *)$ we may assume that $\partial V_{3} \cap W_{i} \neq \emptyset$ for each $i$.

Now the torus boundary $T_{3}$ of $V_{3}$ is contained in $\operatorname{int}\left(W_{i}\right)$ for some $i$. Since $\hat{W}_{i}$ is irreducible we have by Lemma 4 two cases:

(i) $T_{3}$ bounds an (at most once punctured) solid torus $V$ in $W_{i}$

(ii) $T_{3}$ bounds an (at most once punctured) knot space $R$ in $W_{i}$ with a meridian disk $D$ in $\overline{W_{i}-R}$.

Let $Q=W_{i} \cap V_{3}$.

case (i). We replace $W_{i} \cup V_{3}$ in the decomposition for $N \cup V_{3}$ by the (punctured) lens space $L_{l+3}=\overline{W_{i}-Q} \cup_{T_{3}} V_{3}=V \cup_{T_{3}} V_{3}$ (where the union $\cup_{T_{3}}$ is along the common boundary $T_{3}$ ). (See Figure 3 ).
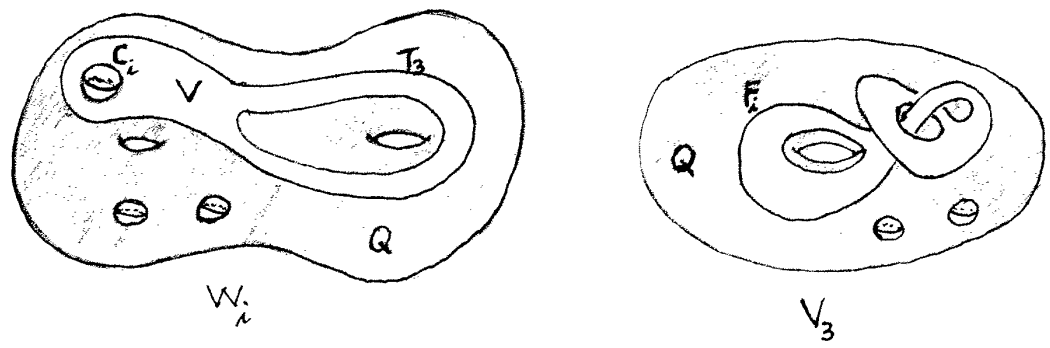

Figure 3: 
Then $M=H \cup K_{1} \cup \cdots \cup K_{m} \cup W_{1} \cup \cdots W_{i-1} \cup W_{i+1} \cup W_{n} \cup L_{1} \cup \cdots \cup L_{l} \cup$ $L_{l+1} \cup L_{l+2} \cup L_{l+3}$ where $F_{j} \subset L_{l+3}$ for all $j \neq i$.

Suppose a 2 -sphere $S$ of $\partial L_{l+3}$ lies in $W_{j}$ for some $j \neq i$. If $S$ bounds a ball in $W_{j}$ let $B$ be an innermost such ball. Then either $L_{l+3} \subset B$ or $L_{l+3} \cap B=\emptyset$. The first case can not happen since $C_{i} \subset L_{l+3}$ and $W_{i} \cap W_{j}=\emptyset$. In the second case replace $L_{l+3}$ by $L_{l+3} \cup B$ in the decomposition of $M$ to get an $L_{l+3}$ with fewer punctures. Hence we may assume that all spheres of $\partial L_{l+3}-C_{i}$ in $W_{j}$ are parallel to $C_{j}$ and we may isotope these 2-spheres across $C_{j}$ out of $W_{j}$ into $H$. Thus we now have $\partial L_{l+3} \cap W_{j}=\emptyset$ and it follows that $W_{j} \subset L_{l+3}$. Deleting $W_{j}$ from the decomposition of $M$ we obtain $M=H \cup K_{1} \cup \cdots \cup K_{m} \cup L_{1} \cup \cdots \cup$ $L_{l} \cup L_{l+1} \cup L_{l+2} \cup L_{l+3}$ with complexity 0 .

Cutting each $K_{j}$ along $S_{j}$ into a (once-punctured) ball $B_{j}$ we obtain a general position decomposition of $M^{\prime}=M \backslash \bigcup_{j} S_{j}$ as in 7(a) with $\partial M^{\prime}$ a collection of 2 -spheres (two copies of $S_{j}$ for each $j$ ). Hence $M$ is obtained from $M^{\prime}=\mathbb{B} \# \hat{L}_{1} \# \cdots \# \hat{L}_{l} \# \hat{L}_{l+1} \# \hat{L}_{l+2} \# \hat{L}_{l+3} \# H_{0}$ by identifying boundary spheres of $H_{0}$ in pairs and we obtain $8\left(d_{1}\right)$.

case (ii). The meridian disk $D$ in $\overline{W_{i}-R}$ is also a meridian disk for $V_{3}$, since $\partial D$ is not null homotopic on $\partial R=T_{3}$. Since $W_{i} \cap W_{j}=\emptyset$ there is a regular neighborhood $U(D)$ in $V_{3}$ that misses $W_{j}$ for all $j \neq i$. In the decomposition of $M=N \cup V_{3}$ replace $W_{i} \cup V_{3}$ by the two punctured balls $B_{0}=R \cup U(D)$ and $B_{1}=\overline{V_{3}-U(D)}$, see figure 4 . Note that $\left(\partial B_{0} \cup \partial B_{1}\right) \cap W_{j}=\emptyset$ for $j \neq i$ and $B_{0} \cup B_{1}=H_{0}$ is a (punctured) ball. As before we can isotope each $W_{j}$
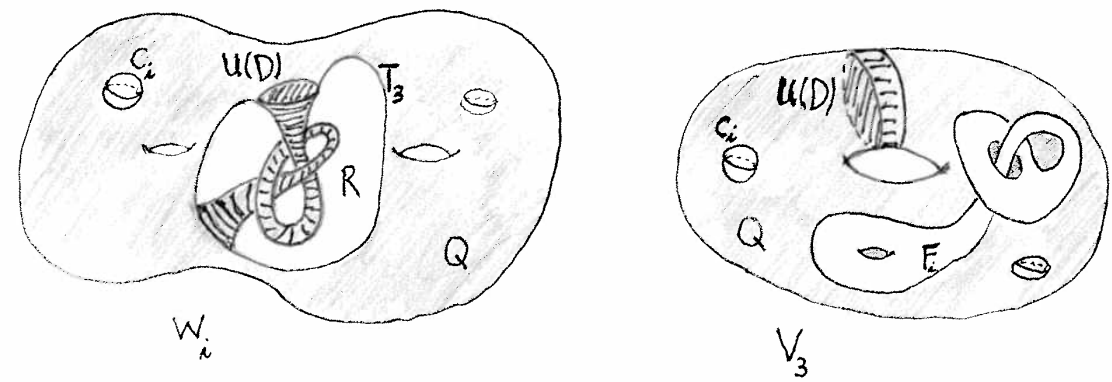

Figure 4:

into $B_{0}$ or $B_{1}$ and then delete the $W_{j}$ 's from the decomposition of $M$ to obtain $M=H \cup K_{1} \cup \cdots \cup K_{m} \cup L_{1} \cup \cdots \cup L_{l} \cup L_{l+1} \cup L_{l+2} \cup H_{0}$ with complexity 0 and proceed as in case (i).

Now consider $\beta \neq 0$. 
Let $c$ be a component of $\mathbf{S}$. If $c \subset \partial V_{3} \cap C_{i}$, say, then $c$ bounds a disk on $C_{i}$ and there is an innermost such disk $D \subset C_{i}$ such that $D \cap \partial V_{3}=\partial D$. Let $U(D)$ be a regular neighborhood rel $\partial V_{3}$ of $D$ in $\operatorname{int}(N)$. If $D \subset V_{3}$ we replace $V_{3}$ by $\overline{V_{3}-U(D)}$ in the decomposition of $M$, if $D$ is not in $V_{3}$ we replace $V_{3}$ by $V_{3} \cup U(D)$. In either case we obtain a new general position decomposition of $M$ with smaller $\beta$ and with $V_{3}$ having one more puncture or $V_{3}$ being replaced by a (punctured) ball $B$ or $V_{3} \cup B$ or a punctured lens space. The result follows now by induction on $\beta$.

In case $7\left(c_{2}\right)$ we represent $N$ as

$$
(* * *) \quad N=H \cup K_{1} \cup \cdots \cup K_{m} \cup W_{1} \cup \cdots \cup W_{n} \cup L_{1} \cup \cdots \cup L_{l} \cup W(S(2))
$$

where $H, K_{j}, W_{i}, L_{k}$ are as before and $W(S(2))$ is now a once punctured Seifert web on $S(2)$. As in the above case $7\left(c_{1}\right)$ we may delete the $W_{j}$ 's from the decomposition $(* * *)$ and assume that $T_{3} \subset \operatorname{int} W(S(2))$.

If $T_{3}$ is compressible in $W(S(2))$ then we repeat the above arguments of cases (i) and (ii) (with $W_{i}$ replaced by $W(S(2)$ ) and noting that $W(S(2))$ is irreducible) to obtain $M$ from $M^{\prime}=\mathbb{B} \# \hat{L}_{1} \# \cdots \# \hat{L}_{l} \# \hat{L}_{l+1} \# H_{0}$ by identifying boundary spheres of the punctured ball $H_{0}$ in pairs. This results in $8\left(d_{1}\right)$.

If $T_{3}$ is incompressible in $W(S(2))$ then $T_{3}$ can be isotoped off the 1-handles into $S(2) \subset W(S(2))$. By Waldhausen's Theorem [W] (see also [Ha]), $T_{3}$ can be further isotoped to consist of fibers.

$$
\text { Let } Q=V_{3} \cap W(S(2)) \text {. }
$$

Suppose there is a component $Q_{1}$ of $Q$ that does not intersect $T_{3}$. Then $Q_{1}$ is a submanifold of $W(S(2))$ such that $\partial Q_{1}$ consists of components of $\partial W(S(2))$. Hence $Q_{1}=W(S(2)) \subset$ int $V_{3}$, and we may delete $W(S(2))$ from $(* * *)$, which leads to $8(\mathrm{a})$.

Thus we now assume that $Q$ is connected. Then $Q$ is a submanifold of $W(S(2))$ such that $\partial Q$ consists of $T_{3}$ and components of $\partial W(S(2))$ and we replace $W(S(2)) \cup V_{3}$ in $(* * *)$ by $W^{\prime}(S(2)) \cup_{T_{3}} V_{3}$, where $W^{\prime}(S(2))=\overline{W(S(2))-Q}$. Since $T_{3}$ is a fibered torus in $S(2) \subset W(S(2))$, the submanifold $W^{\prime}(S(2))$ is again a Seifert web on some $S(2)$. Since $M$ is closed $\partial \hat{W}^{\prime}(S(2))=T_{3}$, hence $\hat{W}^{\prime}(S(2))=S(2)$ and it follows from Proposition 2 of $[\mathrm{H}]$ that $\hat{W}^{\prime}(S(2)) \cup_{T_{3}} \hat{V}_{3}=$ $S(2) \cup_{T_{3}} \hat{V}_{3}$ is either a connected sum of $S^{2}$-bundles over $S^{1}$ (if a fiber of $\partial S(2)$ is homotopic to a meridian of $V_{3}$ ) or an $S(3)$. This results in $8\left(d_{2}\right)$.

Corollary 9 Suppose $M$ is a closed 3-manifold.

(a) If $M$ is a union of three open (punctured) balls then $M \in \mathbb{B}$.

(b) If $M$ is a union of two open (punctured) balls and an open (punctured) solid torus then $M \in \mathbb{B} \# \hat{L}$.

(c) If $M$ is a union of an open (punctured) ball and and two open (punctured) 
solid tori then $M \in \mathbb{B} \# \hat{L}_{1} \# \hat{L}_{2}$.

(d) If $M$ is a union of three open (punctured) solid tori then $\left(d_{1}\right) M \in \mathbb{B} \# \hat{L}_{1} \# \hat{L}_{2} \# \hat{L}_{3}$ or $\left(d_{2}\right) M \in \mathbb{B} \# \tilde{S}(3)$.

\section{Proof.}

By Lemma $1 M$ has a general position decomposition $M=H_{1} \cup H_{2} \cup H_{3}$, resp. $M=H_{1} \cup H_{2} \cup V_{2}$, resp. $M=H_{1} \cup V_{1} \cup V_{2}$, resp. $M=V_{1} \cup V_{2} \cup V_{3}$. Since $\partial M=\emptyset$ the result follows from Theorem 8 .

Remark: It is easy to see that the converse of Corollary 9 is also true. For example, if $M$ is a connected sum of $S^{2}$-bundles over $S^{1}$ and three lens spaces, then we can even decompose $M$ as a union of three solid tori such that their interiors are pairwise disjoint, see e.g. ([GHN], [G]).

\section{References}

[CP] M. Clapp and D. Puppe, Invariants of the Lusternik-Schnirelmann type and the topology of critical sets, Trans. Amer. Math. Soc. 298 (1986), 603-620.

[G] J.C. Gómez-Larrañaga, 3-manifolds which are unions of three solid tori, Manuscripta Math. 59(1987), 325-330.

[GG] J. C. Gómez-Larrañaga and F. González-Acuña, Lusternik-Schnirelmann category of 3-manifolds, Topology 31 (1992), 791-800.

[GGH] J. C. Gómez-Larrañaga, F. González-Acuña, Wolfgang Heil, 3-Manifolds that are covered by two open Bundles, Bol. Soc. Mat. Mexicana (3) Vol. 10, Special issue, 2004

[GGH1] J. C. Gómez-Larrañaga, F. González-Acuña, Wolfgang Heil, A note on Hempel-McMillan coverings of 3-manifolds, Topology Appl. 154 (2007), $1363-1367$

[GGH2] J. C. Gómez-Larrañaga, F. González-Acuña, Wolfgang Heil, Fundamental groups of manifolds with $S^{1}$-category 2, Math. Zeitschrift (2008)

[GGH3] J. C. Gómez-Larrañaga, F. González-Acuña, Wolfgang Heil, Coverings of 3-manifolds by open balls and two open solid tori, preprint.

[GH] J.C. Gómez-Larrañaga and Wolfgang Heil, Seifert unions of solid tori, Math. Z. 240 (2002), no. 4, 767-785.

[GHN] J. C. Gómez-Larrañaga, Wolfgang Heil, V. Nuñez, Stiefel-Whitney surfaces and decompositions of 3-manifolds into handlebodies, Topology Appl. 60 (1994), 267-280 
[H] W. Heil, Elementary surgery on Seifert fiber spaces, Yokohama Math. J. 23, (1974), 135-139.

[Ha] A. Hatcher, Notes on Basic 3-manifold Topology, Lecture Notes, Cornell.

[He] J. Hempel, 3-manifolds, Ann. Math. Studies 86, Princeton Univ Press (1976)

[HM] J. Hempel, D. R. McMillan, Covering three-manifolds with open cells, Fund. Math. 64 (1969), 99-104.

[MT] J. Morgan and G. Tian, Ricci Flow and the Poincarè Conjecture, AMS Clay Math. Monographs, vol 3 (2007).

[W] F. Waldhausen, Eine Klasse von 3-Mannigfaltigkeiten II, Inventiones math. 4, (1967), 87-117. 